\title{
ANÁlise de CARACTERÍSTICA CRÍTICA NO DESENVOLVIMENTO de PRODUTO: INTEGRAÇÃO ENTRE DFMEA E DESENHO DO PRODUTO
}

\author{
Fábio Guedes Correia \\ General Motors do Brasil - Powertrain \\ professor.fguedes@gmail.com
}

\section{RESUMO}

Para tornar seus produtos mais atrativos ao mercado, as organizações vêm focando em lançar novos produtos de forma melhor e com maior número de funcionalidades. Paradoxalmente a essa maior complexidade, as empresas lutam para reduzir o tempo de ciclo de seus projetos para atender com maior eficiência e dinamismo ao mercado cada vez mais exigente e com maior disponibilidade de produtos concorrentes.

Diversas metodologias e ferramentas podem ser aplicadas ao longo do ciclo de vida do produto para atender a essas necessidades, entre elas, destacam-se as ferramentas de qualidade aplicadas ao processo de desenvolvimento de produto e seu processo de manufatura. A aplicação dessas ferramentas no desenvolvimento permite desenvolver um produto certo da primeira vez, no tempo planejado e que satisfaça as expectativas do cliente.

Este estudo tem como principal objetivo propor o uso seqüencial e interdependente de ferramentas para desenvolver um produto e seus processos de manufatura com sucesso, através da correta aplicação da análise de características críticas a serem controladas no processo, considerando as interações entre a análise de modo e efeitos de falhas potenciais no projeto do produto e as especificações e tolerâncias críticas do produto a serem determinadas no desenho do produto.

\section{INTRODUÇÃO}

O aumento da produção, a crescente aplicação da tecnologia, a redução do tempo de ciclo nos projetos e o estabelecimento de novos requisitos de mercado, podem contribuir para o crescente número de falhas durante o desenvolvimento de novos produtos [1] e [2]. Dessa forma, a qualidade de um produto e processo deve ser sempre monitorada, não apenas em relação ao nível de satisfação do cliente, mas também sob os aspectos econômicos que a envolvem [3].

Os aspectos econômicos de qualidade podem ser analisados tanto pelo sucesso ou insucesso nas vendas quanto através de uma análise mais profunda em relação aos investimentos em qualidade e gastos decorrentes da falta dela [3]. No entanto, métodos cada vez mais rigorosos são utilizados para prevenir e mitigar potenciais problemas ao longo do desenvolvimento [4], embora, as ações de prevenção - através do uso de ferramentas de qualidade - nem sempre 
são fáceis de serem mensuradas em relação ao benefício que podem gerar, ao contrário do cálculo obtido com os gastos oriundos dos problemas já ocorridos.

Aplicadas também durante o ciclo de desenvolvimento de um produto, para analisar potencias falhas no projeto e identificar características críticas de controle no processo de manufatura, as ferramentas de qualidade - abordadas neste trabalho - têm como principal objetivo garantir que as especificações de impacto no produto possam ser identificadas, documentadas e comunicadas ao longo de todo o processo, e que os riscos de não atendimento aos requisitos possam ser mitigados.

Devido ao fato de que as características necessárias para o desenvolvimento do processo são oriundas das características do produto [5], as ferramentas abordadas neste trabalho englobam desde a análise das potencias falhas no projeto, através da aplicação da análise de modo e efeitos de falha potencial de projeto (DFMEA), até a identificação das características que necessitam de controle especial no processo de manufatura, através da análise de características críticas. As características críticas identificadas são, então, registradas no desenho do produto, que representa um documento oficial para todo o processo de desenvolvimento e produção, incluindo fornecedores e sistemistas.

Para garantir uma eficiente comunicação ao longo de todo o processo de desenvolvimento do produto, as ferramentas devem ser aplicadas de maneira sequencial e interdependente, de forma que o DFMEA, a análise de características críticas e o próprio desenho do produto, devam ser considerados como fontes de informação para a elaboração e execução do PFMEA, plano de controle e instruções operacionais no posto de trabalho.

\section{PLANEJAMENTO DA APLICAÇÃO DAS FERRAMENTAS DE QUAIDADE}

O planejamento do DFMEA e da análise de características críticas deve ocorrer o quanto antes, dentro do processo de desenvolvimento de novos produtos, e sua execução deve ser iniciada logo após a aprovação do projeto.

O planejamento da aplicação e execução das ferramentas de qualidade deve ser realizado pelo gerente de projeto ou responsável pelo programa, que deve analisar juntamente com os engenheiros envolvidos se haverá necessidade de realizar, por exemplo, um DFMEA de sistema, de subsistema ou simplesmente de componente.

A partir do momento em que existir um detalhamento mais concreto dos componentes de um projeto, obtidos entre as fases de projeto conceitual ou projeto detalhado [1], é possível elaborar uma análise de risco do projeto em relação às ferramentas de qualidade a serem aplicadas. Essa análise deve ser realizada para cada componente do projeto e seu escopo contempla tanto informações do projeto (tabela 1), quanto do desempenho histórico do produto de referência ou baseline (tabela 2).

A aplicação da análise de risco para cada componente do projeto pode auxiliar o time de desenvolvimento a identificar a necessidade de elaboração de, por exemplo, um DFMEA de sistema, subsistema ou apenas de componente, e também de uma análise de características críticas. Caso existam itens considerados como alto risco, o time pode propor a aplicação de ferramentas mais sistêmicas, como o Design for Six Sigma (DFSS) e DFMEA de sistema com diagrama de bloco e matriz de correlação. 
Tabela 1. Modelo de análise de risco do projeto do novo produto.

\begin{tabular}{|c|l|}
\hline \multicolumn{2}{|c|}{ Projeto do novo produto } \\
\hline Tecnologia & $\begin{array}{l}\text { Utilização de uma nova tecnologia ou uma comobinação de tecnologias de um } \\
\text { produtos existentes. Também envolve casos em que uma tecnologia é nova para a } \\
\text { aplicação local, mas já é uma tecnologia aplicada em outra localidade. As saídas } \\
\text { podem ser: Não há nova tecnologia; Existe pouca tecnologia nova; Alto nível de } \\
\text { nova tecnologia. }\end{array}$ \\
\hline $\begin{array}{c}\text { Risco do } \\
\text { design }\end{array}$ & $\begin{array}{l}\text { Nível de modificação de um sistema ou componente. Também inclue se o } \\
\text { programa está em conformidade com o padrão de design e de manufatura } \\
\text { existente. Os resultados podem ser: Baixo risco (produto sem alteração em } \\
\text { relação ao norma; de produção); Risco moderado; Alto risco. }\end{array}$ \\
\hline $\begin{array}{c}\text { Requisitos / } \\
\text { Aplicação }\end{array}$ & $\begin{array}{l}\text { Requisitos e condiçães de operação relacionaddos ao sistema, subsistema e } \\
\text { componentes podem ser didferentes e causar impacto na função. Inclui, por } \\
\text { exemplo, um ítem normal de produção tendo uma nova aplicação ou novas } \\
\text { interfaces. As saídas podem ser: Nenhum requisito novo; Requisito ou aplicação } \\
\text { moderadamente novos; Novo requisito ou aplicação. }\end{array}$ \\
\hline
\end{tabular}

Tabela 2. Modelo de análise de risco em relação ao desempenho histórico do produto referência.

\begin{tabular}{|c|l|}
\hline \multicolumn{2}{|c|}{ Desempenho histórico do produto referência } \\
\hline $\begin{array}{c}\text { Campanhas, } \\
\text { recalls }\end{array}$ & $\begin{array}{l}\text { Referente aos dados de campanha de proutos usados como referência (baseline de } \\
\text { projeto). Os resultados podem ser: Não há; Moderado ; Alto índice de campanhas } \\
\text { de reclamação. }\end{array}$ \\
\hline $\begin{array}{c}\text { Retorno em } \\
\text { garantia }\end{array}$ & $\begin{array}{l}\text { Referente aos dados de garantia de proutos usados como referência (baseline de } \\
\text { projeto). Os resultados podem ser: Não há; Moderado ; Alto índice de } \\
\text { reclamações em garantia. }\end{array}$ \\
\hline $\begin{array}{c}\text { Reclamação } \\
\text { interna }\end{array}$ & $\begin{array}{l}\text { Informações de qualidade, produção e manufatura da organização, em relação } \\
\text { aos proutos usados como referência (baseline de projeto/processo). Os resultados } \\
\text { podem ser: Não há; Moderado ; Alto índice de reclamações internas. }\end{array}$ \\
\hline
\end{tabular}

Independentemente da aplicação ou não da análise de risco, o DFMEA e a análise de características críticas devem ser sempre utilizados no projeto, e a participação de um time multifuncional é importante para melhorar a eficiência da análise, podendo incluir representantes de diversas áreas, como engenharia de produto, manufatura, processo, qualidade do fornecedor, fornecedor, entre outras. 


\section{APLICAÇÃO DO DFMEA}

Também classificado como uma ferramenta de avaliação de risco, o FMEA é uma metodologia analítica utilizada para assegurar que problemas potenciais tenham sido considerados e analisados através do processo de desenvolvimento de um produto ou processo [6]. Originado em 1949 para aplicação militar norte americana, tem sido amplamente utilizado pelo setor da indústria automotiva [7].

O DFMEA requer a participação de um time multifuncional e sua realização no momento certo, ou seja, logo nas fases iniciais do processo de desenvolvimento de um produto, possibilita uma redução de ocorrência de potencias falhas de forma eficiente. Ao longo do desenvolvimento de um produto, o DFMEA pode sofrer diversas revisões, permitindo que as atuais considerações sejam incorporadas no documento.

Os resultados alcançados através do cálculo do Risk Priority Number (RPN) no DFMEA não são recomendados como critério único para análise de necessidade de tomada de ação para mitigação dos riscos [6], sendo uma alternativa bastante eficiente a utilização do critério do Risk Graph Method (RGM) [4] e [8], que não será abordado neste trabalho.

Embora o template de FMEA apresentado pelo manual desenvolvido pela AIAG contenha uma coluna de classificação de característica crítica, o mesmo não demonstra como identificar e classificar tal característica. Dessa forma, a aplicação da análise de características críticas apresentada neste trabalho é uma sugestão de como identificar a necessidade e classificar tais características de forma segura e objetiva.

\section{APLICAÇÃO DA ANÁLISE DE CARACTERÍSTICAS CRÍTICAS}

O principal objetivo da análise de características críticas é a identificação, documentação e comunicação das características que requerem cuidados adicionais e que asseguram conformidade com os requisitos do cliente e regulamentações governamentais, tanto para a organização quanto para seus fornecedores [9].

Intermediando a execução do DFMEA e a definição das características dimensionais ou informativas a serem consideradas no desenho de um produto, a análise de características críticas deveria ser aplicada para todos os componentes de um produto, sendo também essencial a participação de um time multifuncional envolvendo, mas não se limitando, a engenharia de produto, engenharia de manufatura, engenharia de fornecedores, fornecedores, compras, entre outras áreas [9].

É recomendado que todas as características de desenho e de DFMEA sejam migradas para o formulário de análise de características críticas (item 3.1 e 3.2), incluindo sistemas, subsistemas e componentes (SSC), para que não ocorra a chance de que uma característica crítica não seja considerada por esquecimento ou desatenção do time de análise.

Por definição, as características podem ser consideradas como críticas ou não críticas. As não críticas são, geralmente, alvo de requisito de capabilidade para obtenção do processo de aprovação de peças de produção (PPAP), e estabelecem um valor de $\mathrm{Cp} \geq 1,67$ e um $\mathrm{Cpk} \geq$ 1,0 , para a fase de validação do processo de manufatura e produto e, para a fase de produção 
corrente, $\mathrm{Cp} \geq 1,33$ e $\mathrm{Cpk} \geq 1,0$ [10]. No entanto, para as características críticas, o processo se torna mais específico, podendo ser classificadas conforme apresentado na tabela 3.

Tabela 3. Definição e critério das características críticas.

\begin{tabular}{|c|c|c|c|}
\hline $\begin{array}{c}\text { Tipos de Características } \\
\text { Críticas }\end{array}$ & $\begin{array}{c}\text { Descrição das Características } \\
\text { Críticas }\end{array}$ & $\begin{array}{c}\text { Requisitos de } \\
\text { PPAP }\end{array}$ & $\begin{array}{c}\text { Requisitos de } \\
\text { produção corrente }\end{array}$ \\
\hline $\mathrm{CCP}$ & Característica Crítica do Produto & $\begin{array}{c}\mathrm{Cp} \geq 2,0 \\
\mathrm{Cpk} \geq 1,67\end{array}$ & $\begin{array}{r}\mathrm{Cp} \geq 2,0 \\
\mathrm{Cpk} \geq 1,5\end{array}$ \\
\hline $\mathrm{CQP}$ & $\begin{array}{c}\text { Característica de Qualidade do } \\
\text { Produto }\end{array}$ & $\begin{array}{c}\mathrm{Cp} \geq 2,0 \\
\mathrm{Cpk} \geq 1,67\end{array}$ & $\begin{array}{r}\mathrm{Cp} \geq 2,0 \\
\mathrm{Cpk} \geq 1,5\end{array}$ \\
\hline $\mathrm{CDR}$ & $\begin{array}{c}\text { Característica com Documentação } \\
\text { Requerida }\end{array}$ & $\begin{array}{c}\mathrm{Cp} \geq 1,67 \\
\mathrm{Cpk} \geq 1,0\end{array}$ & $\begin{array}{c}\mathrm{Cp} \geq 1,33 \\
\mathrm{Cpk} \geq 1,0\end{array}$ \\
\hline $\mathrm{CAQ}$ & $\begin{array}{c}\text { Característica Atributiva de } \\
\text { Qualidade }\end{array}$ & Não aplicável & Não aplicável \\
\hline
\end{tabular}

As características críticas CCP, CQP e CDR devem atender aos requisitos de capabilidade descritos na tabela 3, onde a documentação de controle estatístico de processo (CEP) deve ser aplicada e mantida com frequiência, preferencialmente em períodos de, no mínimo, uma vez por trimestre para CCP e uma vez por ano para CQP e CDR, a menos que seja necessário aumentar sua frequiência. $\mathrm{O}$ tamanho e freqüência da amostra para estudos estatísticos devem assegurar uma base confiável e representativa para o cálculo da capabilidade, levando em consideração os impactos de causa comum e causa especial no processo. O mesmo controle não deve ser aplicado à característica crítica CAQ, uma vez que seus valores são atributivos, ou seja, aprovado ou não aprovado, por exemplo [10].

Essa análise deve ser aplicada à todos os sistemas, subsistemas e componentes (SSC) completamente novos em relação ao produto corrente; aos SSC's novos, porém com similaridades em relação ao produto atual, cuja modificação tenha impacto direto na função primária do produto; aos SSC's novos, mas com similaridades em relação ao produto atual, cujo histórico pode mostrar problemas de qualidade, custo de garantia, nível de insatisfação do cliente, etc.; e aos SSC's sem modificação e utilizados como "extensão de uso" dentro de um novo projeto, tendo como referência o histórico de qualidade, custo de garantia, nível de insatisfação do cliente, entre outros.

Assim como o DFMEA, sua execução pode ocorrer logo nas fases iniciais do processo de desenvolvimento de um produto e, inclusive, podem coincidir cronologicamente, aproveitando a disponibilidade do time envolvido e as informações disponíveis. Na prática, essas informações serão mais representativas e mais seguras após a definição prévia dos SSC's que comporão o projeto.

\subsection{Análise de características críticas: informações preliminares}

O formulário utilizado como referência foi baseado no template de FMEA da AIAG [6], no qual sugere que o mesmo contenha no mínimo as seguintes informações: 


\subsubsection{Cabeçalho}

Deve conter informações de identificação e rastreabilidade, incluindo número do documento, se a análise refere-se a um sistema, subsistema ou componente, nome do programa, descrição e número do produto, data de elaboração, nome dos profissionais envolvidos na(s) análise(s) e número da revisão do documento.

\subsubsection{Função}

Propósito do item em análise para cumprir o objetivo do projeto.

\subsubsection{Característica}

Nome da variável a ser monitorada, podendo classificar-se entre variável quantitativa e variável qualitativa. Uma variável quantitativa representa algo que se possa mensurar através de instrumentos de medição devidamente calibrados. Exemplos de variáveis quantitativas são o diâmetro interno de um tubo, altura de um corpo, espessura de uma placa, cilindricidade de um eixo, etc.

A variável qualitativa é aplicada a algo que se pretenda verificar o estado de atendimento a um determinado requisito, através de equipamentos ou dispositivos de verificação, não resultando em valores de escala, mas sim em atributo.

Exemplos de varáveis qualitativas são o fato de possuir ou não possuir um furo, altura dentro ou fora do especificado, peça aprovada ou reprovada no teste de estanqueidade, etiqueta correta ou incorreta. Desta forma, uma variável atributiva pode ser utilizada tanto para a verificação do atendimento ou não de uma especificação, como também para a confirmação de presença ou ausência de, por exemplo, uma etiqueta de identificação no produto.

\subsubsection{Dimensão}

É o parâmetro que garante a funcionalidade prevista de um componente, levando em consideração suas interfaces. Suas tolerâncias devem ser sempre consideradas na análise dimensional, pois não existe processo de fabricação isento de desvios ou variações. Além disso, tolerâncias bem definidas garantem a intercambiabilidade das peças. Exemplos de dimensão e suas respectivas tolerâncias são: espessura 5 milímetros podendo variar de \pm 1 décimo de milímetro; altura de $25 \mathrm{~mm} \pm 0,5 \mathrm{~mm}$; comprimento de $150 \mathrm{~mm}+$ $2 \mathrm{~mm}-1 \mathrm{~mm}$.

\subsubsection{Histórico de capabilidade}

Embora todo projeto seja único e diferente, de alguma maneira, de todos os outros produtos e serviços, na maioria das vezes é possível considerar um produto ou um processo como referência, tratando-se as modificações em 
particular. Dessa forma, é coerente considerar a capabilidade atual de processo como referência para uma determinada característica em análise. O time multifuncional deve determinar se existe e qual referência deve ser considerada.

Os valores de referência são utilizados para auxiliar o time a identificar, ainda que previamente, a necessidade de investimentos e esforços para o atendimento de uma determinada característica e sua especificação.

\subsection{Análise de características críticas: fase de execução}

Embora o time multifuncional envolvido na análise deva ter a competência e conhecimentos necessários para definir se uma característica deve ou não ser classificada como crítica e, caso afirmativo e consensual, em qual tipo, uma sequiência de seis questões é apresentada para auxiliar na decisão.

\subsubsection{Existe atualmente um estudo de CEP disponível para essa característica?}

Caso a característica em análise tenha uma referência atual que seja possível obter seus dados de capabilidade do processo de manufatura, disponibilizados em valores de Cp e Cpk, esses valores poderão ser utilizados para avaliar se o processo atual é capaz de alcançar os requisitos necessários quando e se a característica for considerada como crítica. Quando envolve uma característica nova de um SSC o valor de referência para o controle do processo estatístico pode não ser aplicado.

\subsubsection{A redução de variação no processo é essencial para os custos de produção?}

A redução na variação de um processo de manufatura pode impactar em investimentos significativos em custo e tempo e, por isso, sua aplicação deve ocorrer onde realmente existir a necessidade. Um dos motivos que podem justificar a necessidade de reduzir as variações, aumentando os valores de capabilidade, é o impacto dessa variação nos custos de produção.

Nessa questão, a variação é representada por oscilações em torno da média que ocorrem dentro da faixa de limites superior e inferior de especificação. Assim, uma variação significativa - dentro dos limites de especificação pode impactar financeiramente a organização.

\subsubsection{A redução de variação no processo é essencial para a satisfação do cliente?}

Uma variação dentro dos limites superior e inferior de especificação é, geralmente, aceitável, desde que esteja centralizada, com Cpk atendido, e dentro de uma faixa aceitável de variação, com um Cp conforme estabelecido. No entanto, existem casos específicos em que, mesmo estando essa variação dentro de seus limites de especificação, sua oscilação abrupta pode causar impacto significativo no cliente, podendo, este, ser interno ou externo, o governo ou o usuário final do produto. Nesse caso, a satisfação do cliente pode ser prejudicada. 
3.2.4. A redução de variação no processo é essencial para não gerar uma não conformidade?

Também relacionada a uma variação dentro dos limites superior e inferior de especificação, sua oscilação abrupta pode causar impacto significativo no desempenho do produto, gerando uma não conformidade em relação aos requisitos de desempenho estabelecidos. O cliente interno, embora também possa ser considerado o cliente externo, é responsável por avaliar se a variação considerada pode prejudicar o desempenho de um produto. Nessa questão, é necessário interpretar se existe uma grande possibilidade de ocorrência de uma não conformidade se a variação não for reduzida e controlada.

3.2.5. Existe um risco consideráve de impactar o cliente se as peças forem manufaturadas logo fora do limite de especificação?

Existem características que qualquer variação, mínima que seja, fora dos limites de especificação, pode causar um grande impacto negativo ou insatisfação significativa no cliente.

Quando a satisfação do cliente pode ser prejudicada através de um processo com capabilidade mínima aceitável, ou seja, com Cp de 1,33 e um Cpk de 1,0, deve-se considerar a característica como crítica. Para isso, medidas devem ser tomadas para que o processo se torne mais estável e com menor variação em torno da média, reduzindo, assim, a chance de ocorrer um defeito.

3.2.6. Esta é uma característica importante para a função primária do produto e uma variação logo fora do limite de especificação pode causar conseqüências negativas que não sejam significativas?

Existem características que uma variação mínima fora dos limites de especificação pode causar conseqüências negativas de impacto moderado ou insatisfação moderada no cliente. No entanto, a capabilidade de processo com Cp de 1,33 e um Cpk de 1,0 pode ser considerada como aceitável.

\subsection{Resultado do questionário da análise de características críticas}

Baseado nas respostas obtidas em cada item do questionário de identificação de características críticas, é possível determinar se uma característica é considerada crítica ou não pelo time de análise e, caso afirmativo, qual o seu tipo (CCP, CQP, CDR e CAQ).

A tabela 4 apresenta os resultados possíveis de acordo com a resposta de cada questão de identificação de características críticas. 
Tabela 4. Resultado do questionário de identificação de características críticas.

\begin{tabular}{|c|c|c|}
\hline Número da Questão & Resposta da questão & $\begin{array}{c}\text { Tipo de característica resultante do } \\
\text { questionário }\end{array}$ \\
\hline \multirow{3}{*}{3.2 .1} & Sim & variável quantitativa \\
\cline { 2 - 3 } & Não & ----- \\
\hline \multirow{3}{*}{3.2 .2} & Sim & CCP \\
\cline { 2 - 4 } & Não & ----- \\
\hline \multirow{3}{*}{3.2 .3} & Sim & CCP \\
\cline { 2 - 4 } & Não & ----- \\
\hline \multirow{3}{*}{3.2 .4} & Sim & CCP \\
\cline { 2 - 4 } & Não & ------ \\
\hline \multirow{3}{*}{3.2 .5} & Sim & CQP \\
\cline { 2 - 4 } & Não & ----- \\
\hline \multirow{2}{*}{3.2 .6} & Sim & CDR \\
\cline { 2 - 4 } & Não & ----- \\
\hline
\end{tabular}

A afirmação da primeira questão demonstra que - se uma característica de referência possuir controle estatístico aplicado ao processo de manufatura - é possível gerar os valores de capabilidade para a característica em análise, logo, ela pode, seguramente, ser considerada uma variável quantitativa. Sua negação, no entanto, propõe que tal característica seja uma variável atributiva ou que não exista uma referência para se basear.

As questões de número 3.2.2, 3.2.3 e 3.2.4 direcionam a característica para a situação de crítica, classificando-a como CCP, caso qualquer uma das respostas seja positiva. A negação das questões pode sugerir que a característica seja normal (não crítica) ou que a mesma seja crítica classificada como CPQ ou CDR.

A afirmação da quinta e da sexta questões intitula a característica como crítica classificada como CPQ e, respectivamente, como CDR. No entanto, a negação da questão 3.2.5 sugere uma característica CDR ou não crítica, e da sexta e última, apenas não crítica.

\subsection{Detalhamento das características críticas}

\subsubsection{Característica Crítica do Produto $(C C P)$}

Característica onde uma variação notável dentro dos limites de especificação pode afetar significativamente a satisfação do cliente, os custos de produção e/ou o desempenho de um produto. Para essa característica, é requerida evidência documentada e priorizada para a melhoria contínua, na qual seja capaz de originar redução de variação no processo. 


\subsubsection{Característica de Qualidade do Produto (CQP)}

Situação em que o cliente é igualmente satisfeito desde que a característica varie dentro dos limites superior e inferior de especificação. No entanto, o cliente se torna significativamente insatisfeito caso a mesma se encontre imediatamente fora dos valores de especificação.

\subsubsection{Característica com Documentação Requerida (CDR)}

Característica onde uma variação logo fora dos limites de especificação seja improvável afetar significativamente a função ou desempenho de um produto e, conseqüentemente, o cliente. Embora sua capabilidade requerida seja a mesma de uma característica não crítica, faz-se necessário o uso documentado de controle estatístico do processo (CEP).

\subsubsection{Característica Atributiva de Qualidade (CAQ)}

Embora não utilize cálculos para determinação da capabilidade, é considerada crítica pelo fato de requerer um controle de verificação ou confirmação da característica em $100 \%$ dos produtos no processo de manufatura, através de um dispositivo automático à prova de erro. $\mathrm{O}$ controle de verificação pode ser obtido, por exemplo, para controlar uma característica mensurável de forma atributiva. No entanto, o controle de confirmação é mais apropriado para características não mensuráveis, que podem ser, por exemplo, para confirmar a presença de uma etiqueta ou o tipo de material.

Quando não houver a disponibilidade de instrumentos calibrados de medição variável para as características críticas CQP ou CDR, dispositivos atributivos deverão ser utilizados para verificação e controle de uma determinada característica em $100 \%$ da produção, até que o instrumento específico esteja novamente disponível. Nesse período de controle atributivo em $100 \%$ dos produtos, os requisitos de capabilidade estarão suspensos.

Sempre que uma característica apresentar capabilidade abaixo do mínimo requerido, um plano de correção apropriado e documentado deve ser brevemente executado, incluindo uma imediata ação de contenção do produto em relação à característica controlada.

\subsection{Resultado da análise de características críticas e sua interação com o desenho do produto}

Após a realização da análise de todas as características de um produto e a definição das características críticas, o engenheiro do produto é responsável, além da execução das ferramentas aqui apresentadas, por retroalimentar o DFMEA com as características definidas e as informações pertinentes ao produto. Também deve ser responsável por manter os documentos no último nível de revisão e por garantir a comunicação das características entre o time de projeto. 
$\mathrm{O}$ desenho de um produto representa um enorme benefício para transmitir com precisão os detalhes e especificações para que a matéria prima ou componentes possam ser transformados em um produto final. Normalmente, um desenho é realizado por projetistas especializados no domínio das linguagens e normas técnicas de representação, além de manejar com competência os programas apropriados para o desenho técnico.

A elaboração da lista de características críticas e não críticas de um produto é uma entrada para o time de elaboração do desenho do produto, onde as informações de construção, dimensões e tolerâncias serão registradas e oficializadas. Para todas as características críticas definidas, deve existir uma identificação notável que permita a interpretação correta no próprio desenho do produto.

Essa identificação pode ser expressa por uma simbologia, que representa o tipo da característica crítica, ao lado da cota dimensionada. Para uma característica do tipo CAQ, utilizada para confirmação de uma etiqueta ou código de barras, por exemplo, a identificação pode ser descrita no campo de notas de especificação do desenho.

O desenho do produto, o DFMEA e, principalmente, o registro das características críticas definidas devem estar em sincronia, e são fontes importantes para a elaboração consistente do PFMEA e Plano de Controle, que posteriormente transportarão as informações críticas para as folhas de instrução de operação na produção, garantindo que as condições críticas para a qualidade do produto sejam comunicadas a todos que agregam valor ao produto.

\section{CONCLUSÃO}

Aplicar a análise de características críticas na fase inicial do desenvolvimento de um produto, especificamente na fase de execução do DFMEA, permite um maior detalhamento e melhor entendimento da necessidade de controle de uma característica crítica do produto no processo de manufatura. Sua realização em conjunto com o DFMEA também permite aproveitar a interação e sinergia do time de projeto para uma análise mais completa e consistente.

Quando não existe uma análise profunda e sistematizada da necessidade de uma característica crítica, é provável que haja desperdício de recursos financeiros e esforços para uma característica que não seja tão impactante para o desempenho de um produto. No contrário, e com consequiências ainda mais agravantes para a organização e sua cadeia produtiva, pode uma característica de impacto significativo para o desempenho do produto não ser tratada com seus devidos cuidados, proporcionando uma probabilidade considerável de insatisfação no cliente.

Além disso, os registros da análise de características críticas podem ser considerados documentos importantes para a prática de lições aprendidas, quando sua recuperação pode disponibilizar informações históricas de conhecimento técnico em relação ao projeto atual ou até mesmo aos projetos futuros. 
A análise de características críticas suporta a integração e a comunicação entre o DFMEA e o desenho do produto e, por consequência, agrega no conjunto de informações que deverão ser utilizadas para alimentar o PFMEA, plano de controle e, respectivamente, as instruções de operação, de forma dinâmica e eficaz.

\section{REFERÊNCIAS}

[1] ROZENFELD, H. et al. Gestão de desenvolvimento de produtos: uma referência para a melhoria do processo. São Paulo: Saraiva, 2006.

[2] CORREIA, F. G. O processo de desenvolvimento de novos produtos: um estudo de caso no setor automotivo. 2012. 102 f. Dissertação (Mestrado em Engenharia Mecânica) - FEI, São Paulo, 2012.

[3] MIGUEL, P. A. Cauchick. Qualidade: enfoques e ferramentas. São Paulo: Altliber, 2001. [4] CORREIA, F. G.; COLLINI, C. P. Análise de Modo e Efeitos de Falha - FMEA: aplicação do Risk Graph Method como critério de análise de risco. In: CONGRESSO SAE BRASIL 2012. ago. 2012, São Paulo. Anais... São Paulo: SAE Brasil International, 2012. p. 1.

[5] JURAN, J. M. A qualidade desde o projeto - novos passos para o planejamento da qualidade em produtos e serviços. São Paulo: Pioneira Thomson Learning, 2004.

[6] AIAG. Potential Failure Mode and Effect Analysis. 4. ed. [S.1.]: AIAG, Jul. 2008.

[7] FRITZSCHE, R. Failure Mode and Effects Analysis (FMEA): A comparison between VDA-Approach versus QS-9000, SAE International, 2011.

[8] CORREIA, F. G. Análise de risco no processo de desenvolvimento de produto: método gráfico e critério de RPN. In: CONGRESSO SIMEA 2012. set. 2012, São Paulo. Anais... São Paulo: Associação Brasileira de Engenharia Automotiva, 2012. p. 1.

[9] CORREIA, F. G. Aplicação de ferramentas de qualidade no desenvolvimento de produto: DFMEA e análise de características críticas de controle no processo de manufatura. In:

CONGRESSO SAE BRASIL 2012. ago. 2012, São Paulo. Anais... São Paulo: SAE Brasil International, 2012. p. 1.

[10] BAIDU. GM 1927-03 SQ SOR. Supplier Quality Statement of Requirements. Disponível em: <http:// wenku.baidu.com/view/6b23d73210661ed9ad51f317>. Acesso em: 10 mai. 2013. 